\title{
Intellectual disability and microcephaly associated with a novel CHAMP1 mutation
}

\author{
Yuta Asakura ${ }^{1}$, Hitoshi Osaka $\mathbb{D}^{1 凶}{ }^{凶}$, Hiromi Aoi $^{2}$, Takeshi Mizuguchi ${ }^{2}$, Naomichi Matsumoto ${ }^{2}$ and Takanori Yamagata ${ }^{1}$
}

(C) The Author(s) 2021

Mutations in a number of genes related to chromosomal segregation reportedly cause developmental disorders, e.g., chromosome alignment-maintaining phosphoprotein 1 (CHAMP1). We report on an 8-year-old Japanese girl who presented with a developmental disorder and microcephaly and carries a novel nonsense mutation in CHAMP1. Therefore, CHAMP1 mutation should be considered as a differential diagnosis of global developmental delay and microcephaly.

Human Genome Variation (2021) 8:1-3; https://doi.org/10.1038/s41439-021-00165-7

Chromosome alignment-maintaining phosphoprotein 1 (CHAMP1) is a zinc finger protein that functions in kinetochore-microtubule attachment and regulates chromosome segregation. Pathogenic variants of CHAMP1 have been reported in patients with intellectual disability and other signs, such as microcephaly, muscular hypotonia, facial dysmorphism, and eye anomalies. ${ }^{1-3}$ Here, we report a novel nonsense mutation in CHAMP1, NM_001164144.2:c.1465C>T, p.(Gln489*).

The patient was an 8-year-old Japanese girl born to nonconsanguineous parents without neurological abnormalities, such as intellectual disability. She was delivered at full term by emergency Cesarean section because of umbilical cord entanglement without newborn asphyxia. Her birth weight was $2755 \mathrm{~g}$ $(-0.47$ standard deviation [SD]), her length was $46.8 \mathrm{~cm}(-1.5 \mathrm{SD})$, and her occipital frontal circumference was $31.8 \mathrm{~cm}(-0.9$ SD). Her physical examination at birth was normal. She could control her head at 4 months of age and sit stably at 10 months. At 10 months of age, she developed acute encephalopathy after infection with respiratory syncytial virus and was treated with steroid pulse therapy in another hospital. She recovered without sequelae. However, since her recovery, developmental delay with acquired microcephaly $(-2.4 \mathrm{SD})$ has become evident. Head magnetic resonance imaging was performed when she was 2 years old, and the scan showed mild atrophy of the cerebrum and cerebellum. She could walk independently at 3.5 years of age.

At 4 years of age, she came to our hospital because of global intellectual disability and acquired microcephaly. At the time of the visit, she had severe intellectual disability and could speak no meaningful words. A physical examination revealed no abnormalities. She was very friendly and displayed no dysmorphic facial features. A neurological examination showed normal muscle tone and tendon reflexes. No pyramidal tract signs, dystonia, or involuntary movements were observed. Her intelligence quotient according to a Tanaka-Binet test was 35 . As the acute encephalopathy caused no brain magnetic resonance imaging abnormalities or sequelae, we searched for the cause of her intellectual disability and microcephalus. We initially suspected Angelman syndrome; however, a fluorescence in situ hybridization analysis failed to show a microdeletion at 15q11.2. After receiving informed consent, we performed whole-exome sequencing as previously described. ${ }^{4}$ We identified a novel de novo nonsense heterozygous variant, c.1465C>T, p.(GIn489*), in CHAMP1 (Fig. 1).

In addition to $C H A M P 1,{ }^{1-3}$ a number of mutations in genes related to chromosome alignment and/or spindle function, including Pogo transposable element-derived with ZNF domain $(P O G Z)$, tubulin gamma 1, dynein cytoplasmic 1 heavy chain 1 , kinesin family member 5C (KIF5C), KIF2A, KIF4A, and centromere protein $E$, have been shown to cause various developmental disorders. $^{5-9}$ CHAMP1 contains several repeat motifs, i.e., SPE, WK, and FPE motifs, and regulates kinetochore-microtubule attachment and chromosome alignment (Fig. 1). These motifs reportedly play an important role in spindle localization and kinetochore-microtubule interactions. ${ }^{10}$ The C-terminal region of CHAMP1, which contains the zinc-finger domain, has been predicted to be crucial for its proper localization to chromosomes and for mitotic spindle function. ${ }^{1}$ Functional studies using various deletion mutants of CHAMP1 have shown that lack of the C-terminal region of CHAMP1 prevents proper chromosomal localization. ${ }^{10}$ Similar to the CHAMP1 mutation in this case, all reported mutations are truncating mutations, i.e., nonsense or frameshift mutations that cause the loss of the C-terminal zinc-finger domain (Table 1). Although the biological mechanism that links CHAMP1 mutations and intellectual disability is unknown, the pathogenic mechanism appears to be a result of the loss of the C-terminal region of CHAMP1. POGZ binds to the C-terminal region of CHAMP1 and is critical for proper chromosome segregation. ${ }^{3,11}$ Therefore, loss of the C-terminus may impair CHAMP1 chromosomal localization or/ and its binding to other proteins, such as POGZ. Mitotic defects in neural progenitor cells may cause a decrease in the number of neural cells and defective neural development, resulting in intellectual disability. ${ }^{3}$

\footnotetext{
'Department of Pediatrics, Jichi Medical University, Tochigi, Japan. ${ }^{2}$ Department of Human Genetics, Yokohama City University Graduate School of Medicine, Yokohama, Japan. 更email: hosaka@jichi.ac.jp
}

Received: 22 May 2021 Revised: 5 July 2021 Accepted: 8 July 2021

Published online: 17 August 2021 


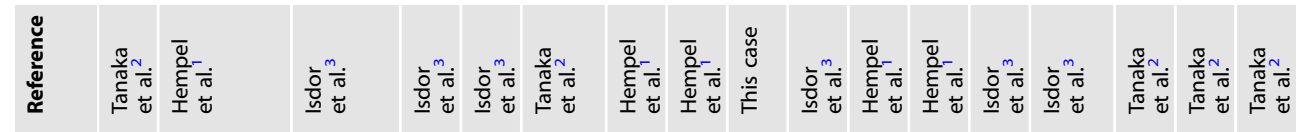
Un

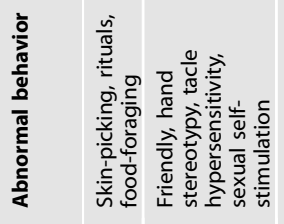

1

$+\frac{s}{z}++1+++++\frac{\pi}{z} \frac{\pi}{z} \frac{\pi}{z}$

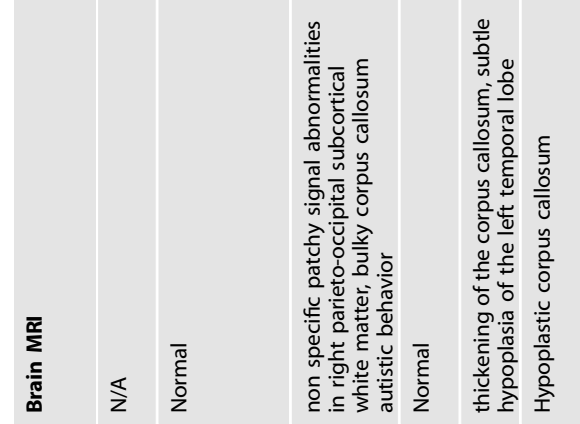

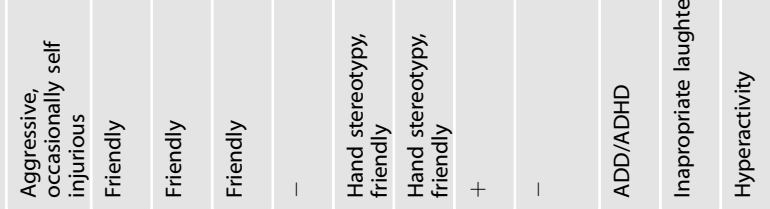
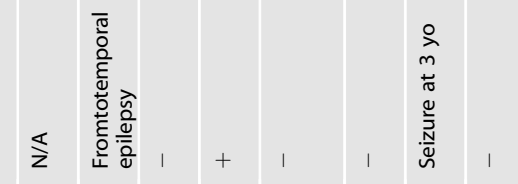

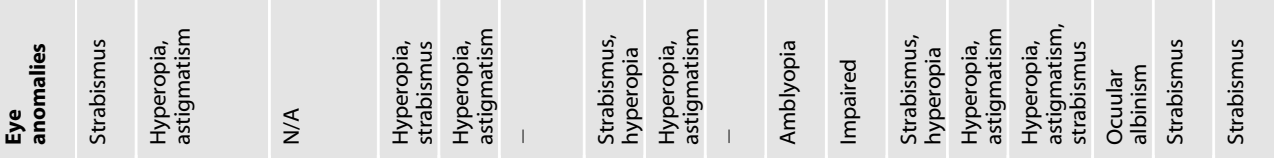

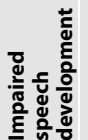

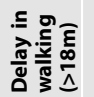

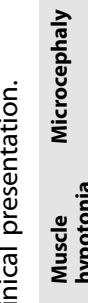

$\frac{\sqrt{0}}{\frac{\pi}{\pi}}$

$\stackrel{2}{\longleftarrow}$

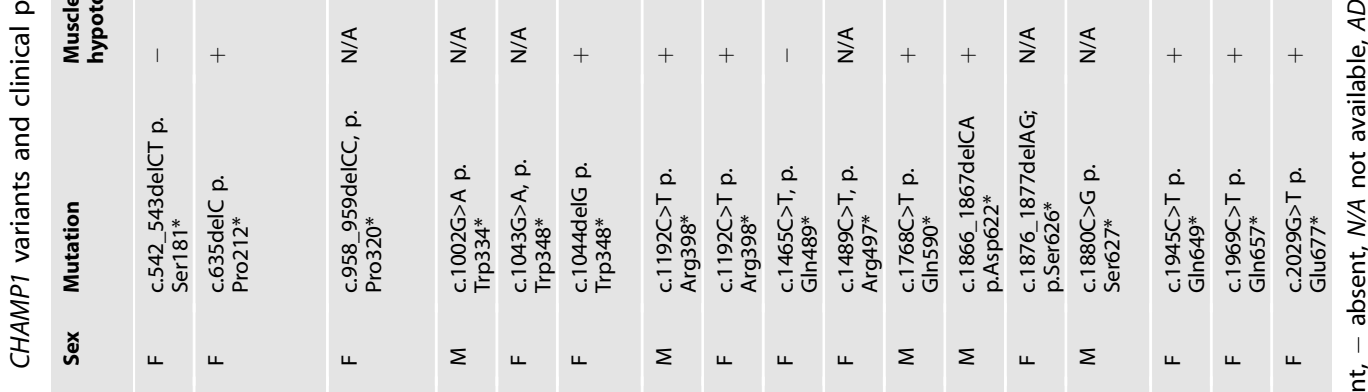

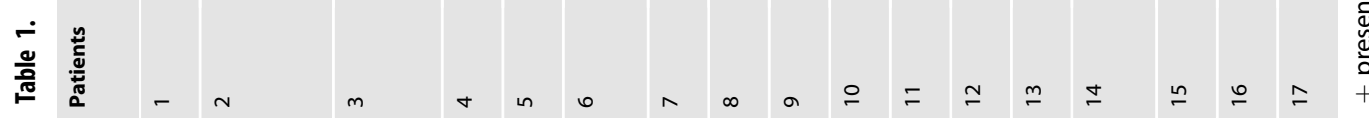




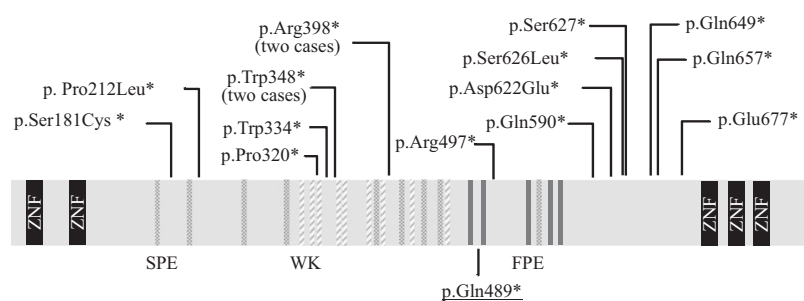

Fig. 1 Diagram of CHAMP1 variants. Diagram of previously reported CHAMP1 variants (upper panel) and the mutation identified in this case (lower panel, underlined). CHAMP1 consists of five zinc-finger domains (ZNF) and several motifs; SPE (consensus: PxxSPExxK; dots), WK (SPxxWKxxP; diagonal lines), and FPE (FPExxK; grey bar).

The clinical presentation of CHAMP1 mutation includes intellectual disability (17/17), motor development delay (14/17), facial dysmorphism (10/12), eye anomalies (14/16), and microcephaly $(12 / 17)$. For some individuals, anomalies are evident on magnetic resonance imaging scans of the brain (9/15) and abnormal behavior varies from mild to severe $(14 / 17)$ (Table 1). ${ }^{1-3}$ Some patients present with a feeding disorder. Considering these symptoms, Angelman syndrome and Prader-Willi syndrome are the most important differential diagnoses. ${ }^{1}$

In conclusion, we report a novel nonsense mutation in CHAMP1, c. $1465 C>T$, p.(Gln489*). The patient had intellectual disability, motor development delay, and microcephaly but no facial dysmorphism or eye anomalies. CHAMP1 mutation should be considered when a patient presents with global developmental delay and microcephaly.

\section{HGV DATABASE}

The relevant data from this Data Report are hosted at the Human Genome Variation Database at https://doi.org/10.6084/m9. figshare.hgv.3081.

\section{REFERENCES}

1. Hempel, M. et al. De novo mutations in CHAMP1 cause intellectual disability with severe speech impairment. Am. J. Hum. Genet. 97, 493-500 (2015).

2. Tanaka, A. J. et al. De novo pathogenic variants in CHAMP1 are associated with global developmental delay, intellectual disability, and dysmorphic facial features. Cold Spring Harb. Mol. Case Stud. 2, 1-8 (2016).

3. Isidor, B. et al. De novo truncating mutations in the kinetochore-microtubules attachment gene CHAMP1 cause syndromic intellectual disability. Hum. Mutat. 37, 354-358 (2016)

4. Aoi, $\mathrm{H}$. et al. Whole exome sequencing of fetal structural anomalies detected by ultrasonography. J. Hum. Genet 66, 499-507 (2021).

5. Fitzgerald, T. W. et al. Large-scale discovery of novel genetic causes of developmental disorders. Nature 519, 223-228 (2015).
6. Poirier, K. et al. Mutations in TUBG1, DYNC1H1, KIF5C and KIF2A cause malformations of cortical development and microcephaly. Nat. Genet 45, 639-647 (2013).

7. Willemsen, M. H. et al. Involvement of the kinesin family members KIF4A and KIF5C in intellectual disability and synaptic function. J. Med. Genet 51, 487-494 (2014).

8. Mirzaa, G. M. et al. Mutations in CENPE define a novel kinetochore-centromeric mechanism for microcephalic primordial dwarfism. Hum. Genet 133, 1023-1039 (2014).

9. Hanks, S. et al. Constitutional aneuploidy and cancer predisposition caused by biallelic mutations in BUB1B. Nat. Genet. 36, 1159-1161 (2004).

10. Itoh, G. et al. CAMP1 (C13orf8, ZNF828) is a novel regulator of kinetochoremicrotubule attachment. EMBO J. 30, 130-144 (2011).

11. Nozawa, R. et al. Human POGZ modulates dissociation of HP1a from mitotic chromosome arms through Aurora B activation. Nat. Cell Biol. 12, 719-727 (2010).

\section{ACKNOWLEDGEMENTS}

This work was supported in part by the Japan Agency for Medical Research and Development (AMED) under Grant numbers JP20ek0109486, JP21ek0109549, JP21 cm0106503, and JP21ek0109493 (N.M); the Japan Society for the Promotion of Science (JSPS) KAKENHI (Grant number JP20K08164 (T.M.); and the Takeda Science Foundation (T.M.).

\section{COMPETING INTERESTS}

The authors declare no competing interests.

\section{ADDITIONAL INFORMATION}

Correspondence and requests for materials should be addressed to H.O.

Reprints and permission information is available at http://www.nature.com/ reprints

Publisher's note Springer Nature remains neutral with regard to jurisdictional claims in published maps and institutional affiliations.

(i) Open Access This article is licensed under a Creative Commons Attribution 4.0 International License, which permits use, sharing, adaptation, distribution and reproduction in any medium or format, as long as you give appropriate credit to the original author(s) and the source, provide a link to the Creative Commons license, and indicate if changes were made. The images or other third party material in this article are included in the article's Creative Commons license, unless indicated otherwise in a credit line to the material. If material is not included in the article's Creative Commons license and your intended use is not permitted by statutory regulation or exceeds the permitted use, you will need to obtain permission directly from the copyright holder. To view a copy of this license, visit http://creativecommons. org/licenses/by/4.0/.

(c) The Author(s) 2021 